\title{
Crianças Portadoras de Leucemia Linfóide Aguda: Análise dos Limiares de Detecção dos Gostos Básicos
}

Acute Lymphocytic Leukemia in Children: Analysis of Detection Thresholds for Basic Tastes According to Gender

\author{
Ilana Elman, Maria Elisabeth Machado Pinto e Silva*
}

\section{Resumo}

Considera-se câncer, na infância, toda neoplasia maligna que acomete indivíduos menores de 15 anos. A leucemia é o tipo mais comum nesta população e a linfóide aguda (LLA) é mais comum em meninos do que em meninas, numa proporção de $(1,2: 1)$. Aproximadamente $50 \%$ dos pacientes com câncer reportam anormalidades no comportamento alimentar por redução no apetite, dificuldades mecânicas, alterações no paladar, náuseas, vômitos, diarréias, dentre outras. A determinação dos limiares de detecção dos gostos básicos contribui para compreender as aversões e as preferências alimentares das crianças portadoras de câncer. O objetivo do estudo foi identificar os limiares de detecção para os gostos básicos, por sexo, de crianças portadoras de LLA em tratamento quimioterápico. Participaram crianças de três instituições desta especialidade em São Paulo: ITACI/ICR/HC/FMUSP, IOP GRAAC e o Hospital A.C. Camargo. A pesquisa foi realizada envolvendo 40 crianças, de 6 a 15 anos, sendo 21 do sexo masculino e 19 do feminino, que seguiam o protocolo de tratamento GBTLI-99. Aplicou-se o teste de sensibilidade de Threshold, utilizando amostras pareadas, em séries crescentes de seis concentrações distintas para cada gosto. Foram aplicados testes não-paramétricos, utilizando-se o programa SPSS. A variável sexo não mostrou diferenças significativas em relação à análise dos limiares de detecção dos gostos básicos.

Palavras-chave: Criança, Leucemia, Limiar, Sexo

*Departamento de Nutrição - Faculdade de Saúde Pública - Universidade de São Paulo (USP) - São Paulo (SP) Endereço para correspondência: Ilana Elman. Departamento de Nutrição - Faculdade de Saúde Pública / USP. Av. Dr. Arnaldo, 715 Cerqueira César São Paulo (SP) - CEP: 01246-904. E-mail: ilanae@usp.br 


\section{INTRODUÇÃO}

Câncer na infância é considerado como toda neoplasia maligna que acomete aqueles indivíduos menores de 15 anos $^{1}$. Estima-se uma incidência anual de cerca de 200 mil casos em todo o mundo ${ }^{2}$, sendo a leucemia o tipo mais comum nesta população ${ }^{3}$.

Leucemias agudas são neoplasias primárias de medula óssea caracterizadas por formarem um grupo heterogêneo de doenças, nas quais existe a substituição dos elementos medulares e sangüíneos normais por células imaturas ou diferenciadas denominadas blastos, bem como acúmulo destas células em outros tecidos. A leucemia linfóide aguda possui bom prognóstico, com $95 \%$ de remissão completa em casos tratados com quimioterapia ${ }^{1}$. Incidem na população de 0 a 14 anos, em uma freqüência de 1/25.000 indivíduos/ano e o risco de desenvolver a doença nos primeiros 10 anos é de $1 / 2.880$. A LLA é mais comum em crianças brancas do que negras $(1,8: 1)$, e em meninos do que meninas $(1,2: 1)^{4}$. A etiologia ainda não está determinada, embora sejam enfatizados como possíveis causas: efeitos da irradiação, exposição a drogas antineoplásicas, fatores genéticos associados, imunológicos e exposição a alguns vírus ${ }^{5}$.

O tratamento da LLA é prolongado, variando de dois a três anos. Embora os esquemas terapêuticos possam mudar entre os diversos centros, os protocolos modernos invariavelmente são constituídos de cinco fases: indução de remissão, intensificação-consolidação, reindução, prevenção da leucemia no sistema nervoso central e continuação ou manutenção de remissão $0^{4}$.

No Brasil, na década de 1980, deu-se início ao primeiro protocolo brasileiro multicêntrico de tratamento da LLA infantil, formando-se assim o Grupo Cooperativo Brasileiro de Tratamento de Leucemia Linfóide Aguda na Infância (GBTLI-LLA-80). Desde então, três estudos multicêntricos foram realizados e concluídos $(1982,1985,1993)$. A partir dos resultados, observou-se uma crescente possibilidade de cura para a criança portadora de LLA no Brasil, com curvas de sobrevida livre de eventos para todos os grupos de risco que saíram de 50\% no GBTLI-LLA-80 para índices de $70 \%$ no GBTLI-LLA-93 $3^{5,6}$.

Todos estes protocolos adotaram, como critério de risco, os dados clínico-laboratoriais pré-tratamento, adaptando a intensidade da quimioterapia e da radioterapia aos diferentes grupos, sendo aperfeiçoados continuamente. Como exemplo, há o Protocolo GBTLI LLA-99, o qual é baseado na experiência dos resultados dos estudos LLA-80, 82, 85 e $93^{7}$.

Medicamentos utilizados no Protocolo GBTLI-99 possuem efeitos variados no organismo, como transtornos gastrintestinais, diminuição do apetite, sensação do gosto metálico, dentre outros ${ }^{8}$.

Embora haja efeitos colaterais, pelo conjunto dessas açôes, a maioria das crianças portadoras de neoplasias é tratada com vistas à cura 9 . Atualmente, cerca de $70 \%$ a $75 \%$ das crianças podem ser curadas com os protocolos de tratamento atuais ${ }^{10}$.

Tem-se observado, na população infantil portadora de câncer, reduzida ingestão calórica e proteica nas diversas fases da doença, por redução no apetite, dificuldades mecânicas, alterações no paladar, náuseas, vômitos, diarréias, e jejuns prolongados para exames pré ou pósoperatórios, decorrentes da quimioterapia e da radioterapia. Estudos avaliaram que, durante o ciclo da quimioterapia, crianças e adolescentes com câncer apresentaram redução de $40 \%$ a $50 \%$ na ingestão habitual ${ }^{11,12}$.

Os tratamentos, principalmente a quimioterapia e a radioterapia, têm efeitos agressivos para o hospedeiro, por deixar o organismo vulnerável e debilitado, aumentar o risco para o comprometimento nutricional e prejudicar a resposta terapêutica ${ }^{13}$.

Normalmente, crianças com alguma doença podem ter seu apetite alterado e conseqüentemente afetar seu balanceamento em nutrientes ${ }^{14}$. Isto se deve à existência de aversões alimentares e inapetência durante o tratamento antineoplásico, o que contribui para uma depleção nutricional muito mais intensa ${ }^{15}$.

As qualidades sensoriais (sabor, odor, textura e aparência) são fatores determinantes do comportamento alimentar e desempenham um papel não somente na determinação de seu consumo, como também da saciedade, ingestão e seleção do alimento numa refeição ${ }^{16}$.

As crianças aprendem a associar os estímulos do gosto dos alimentos às conseqüências fisiológicas à sua ingestão. Um exemplo desse tipo de aprendizagem é a aversão condicionada resultante do comer um alimento que provoca conseqüências negativas a seguir, como náuseas e vômitos ${ }^{17}$. Este fato associado aos efeitos causados pelos tratamentos antineoplásicos pode explicar a diminuição da aceitação alimentar dos pacientes.

Os tratamentos antineoplásicos utilizados, a quimioterapia e a radioterapia reduzem a produção de saliva causando a xerostomia, sendo que, conseqüentemente, a percepção do sabor dos alimentos também se altera ${ }^{18}$.

Os deficits da gustação não apenas reduzem o prazer e o conforto provenientes dos alimentos, mas são causa de sérios fatores de risco para as deficiências nutricionais e imunológicas. As pessoas com deficiência na percepção do sabor fazem suas escolhas dos alimentos com base no que lhes atrai, sem considerar seu valor nutricional, o que dificulta a adesão a regimes dietéticos específicos ${ }^{19}$. 
Para medir, analisar e interpretar reações das características dos alimentos, como são percebidos pelos órgãos da visão, olfato, gosto, tato e audição faz-se uso da análise sensorial, uma disciplina científica ${ }^{20}$.

Uma das ferramentas da análise sensorial é o teste de sensibilidade ou Threshold, que pela sua especificidade proporciona subsídios para a formulação de produtos direcionados a cada tipo de consumidor. Tais testes medem a habilidade de perceber, identificar e/ou diferenciar um ou mais estímulos pelos órgãos dos sentidos ${ }^{21-24}$.

O índice de limiar (Threshold) depende da intensidade do estímulo causado pela concentração da solução, podendo ser classificado em limiar absoluto, reconhecimento, diluição e detecção. No teste de limiar de detecção, a intensidade mínima detectável é medida pelo estímulo referido pelo provador, mas sem caracterizá-lo, por exemplo, não expressar se o gosto analisado é doce ou salgado ${ }^{21,25}$.

Estudos desenvolvidos com crianças de 8 e 9 anos de idade mostraram resultados que indicam diferenças encontradas entre os sexos para a sensibilidade dos gostos entre meninos e meninas, porém sugerem que essas diferenças sejam transitórias ${ }^{26}$.

Propõe-se que crianças são menos sensíveis ao gosto doce do que os adultos, sendo assim, para investigar os níveis do gosto doce que as crianças são capazes de discriminar, testes sensoriais confiáveis são necessários ${ }^{27}$.

Caratin $^{28}$ realizou o teste de limiar para os gostos básicos em escolares saudáveis, de 7 a 10 anos, onde se observou que o sexo masculino apresentou maior sensibilidade ao gosto amargo e azedo, enquanto as meninas apresentaram maior sensibilidade ao gosto ácido e doce.

A determinação dos limiares de detecção dos gostos básicos contribui para compreender as aversōes e as preferências alimentares das crianças portadoras de câncer, melhorando a alimentação e contribuindo para a recuperação do estado nutricional com consumo alimentar adequado. Uma vez que esse grupo pode apresentar grande dificuldade em se alimentar, devido à doença e suas complicações, todos os testes que identifiquem a sensibilidade e que, conseqüentemente, influenciarão na orientação e aceitação alimentar devem ser realizados.

O objetivo desta pesquisa foi identificar os limiares de detecção para os gostos básicos, por sexo, de crianças portadoras de LLA em tratamento quimioterápico.

\section{METODOLOGIA}

\section{POPULAÇÃO EM ESTUDO}

Foram avaliados os pacientes pediátricos de 6 a 15 anos de idade, em tratamento antineoplásico, com diagnóstico de leucemia linfóide aguda (LLA), seguindo o protocolo GBTL-99. Participaram crianças de três instituiçôes desta especialidade, em São Paulo: Instituto de Tratamento do Câncer Infantil (ITACI) do Instituto da Criança do Hospital das Clínicas de São Paulo; Hospital do Câncer A.C. Camargo e Instituto de Oncologia Pediátrica - Grupo de Apoio à Criança e Adolescente com Câncer (GRAAC).

\section{CRITÉRIOS DE EXCLUSÃo}

Foram excluídas as crianças que apresentaram febre, gripe, resfriado ou alguma complicação na cavidade bucal, pois estes fatores podem interferir na percepção dos gostos. Também foram excluídas aquelas que não apresentavam condiçôes de se expressar.

\section{ANÁLISE SENSORIAL: LOCAL DE REALIZAÇÃO DOS TESTES DE SENSIBILIDADE}

Os testes foram realizados na própria instituição, individualmente, em local isento de ruídos e odores, temperatura agradável e luz natural ${ }^{23}$.

\section{Preparo das amostras para 0 teste do limite}

As amostras foram preparadas no Laboratório de Bromatologia do Departamento de Nutrição da Faculdade de Saúde Pública da USP, utilizando água deionizada para as soluçôes e balança analítica para quantificar os solutos utilizados: sacarose, cloreto de sódio, cafeína, ácido cítrico ${ }^{29}$. As soluçōes, preparadas quinzenalmente, foram acondicionadas em garrafas plásticas de $250 \mathrm{ml}$, devidamente identificadas e armazenadas em temperatura ambiente e local arejado nas instituiçóes de coleta de dados.

Foi utilizado o teste de sensibilidade de Threshold, o qual mede a habilidade de perceber, identificar e/ou diferenciar um ou mais estímulos pelos órgãos dos sentidos ${ }^{21,25}$. As concentraçôes utilizadas foram as preconizadas pela International Organization for Standartization ${ }^{29}$ para a determinação dos gostos, adaptadas por Caratin ${ }^{28}$, as quais foram consideradas eficientes na determinação dos gostos básicos. As concentraçóes dos solutos por litro de água deionizada foram: 0,$500 ; 1,000 ; 2,000 ; 4,000 ; 8,000$ e 16,000 para o doce; 0,$090 ; 0,180 ; 0,370 ; 0,750 ; 1,500$ e 3,000 para o salgado; 0,$004 ; 0,008 ; 0,015 ; 0,030 ; 0,062$ e 0,125 para o ácido; 0,$025 ; 0,050 ; 0,100 ; 0,200 ; 0,400$ e 0,800 para o gosto amargo.

\section{APLICAÇÃO DO TESTE DOS LIMIARES}

As amostras foram apresentadas aos pares (solução e água deionizada), em séries crescentes de seis concentraçōes distintas para cada gosto, cabendo ao provador indicar se algum estímulo tivesse sido detectado. 
Para que a criança compreendesse como proceder durante o teste, foi oferecida uma solução-padrão (água deionizada) e uma outra solução, e a criança deveria informar se as amostras pareadas eram iguais (nãodetecção) ou diferentes (detecção, desde que seja apontada corretamente a solução). A apresentação das soluções continuou até que as crianças detectassem algum estímulo duas vezes consecutivo, cessando a seqüência. Repetiu-se a apresentação, partindo da solução de menor concentração do soluto (teste em duplicata). Após a avaliação de cada par de amostras, a criança foi instruída a lavar as papilas com água e aguardar cerca de 30 segundos para provar o próximo par.

Em nenhum momento foi solicitada a identificação do gosto testado, sendo que a ordem apresentada foi a mesma para todas as crianças (doce, salgado, ácido e amargo), uma vez que o objetivo foi o de verificar o limiar de sensibilidade para os mesmos.

\section{ANÁLISE ESTATÍSTICA}

Utilizaram-se testes não-paramétricos, uma vez que as variáveis não possuíam distribuição normal. Para a análise dos dados, utilizou-se o pacote estatístico SPSS versão 10.031 .

A comparação dos limiares (média aritmética das duas medidas realizadas), por sexo, foi feita pelo teste de Mann-Whitney ${ }^{32}$.

\section{QUESTÕES ÉTICAS}

Este trabalho foi submetido e aprovado pelo Comitê de Ética em Pesquisa da Faculdade de Saúde Pública da Universidade de São Paulo e pelos demais comitês das instituiçōes envolvidas na pesquisa.

As instituiçōes em que este trabalho foi realizado foram consultadas sobre as condiçōes para desenvolvêlo e assinaram um termo de consentimento segundo os moldes da resolução no ${ }^{\circ} 196$ de 10/10/1996 do Conselho Nacional de Saúde, após a submissão e aprovação desta pesquisa pelos seus comitês de ética.

Os responsáveis foram informados e esclarecidos sobre os objetivos e metodologia do estudo e autorizaram a participação da criança, assinando um termo de consentimento segundo a resolução no ${ }^{\circ} 196$ de 10/10/1996 do Conselho Nacional de Saúde. Só participaram do estudo as crianças que entregaram a autorização.

\section{RESULTADOS E DISCUSSÃO}

\section{Caracterizaç̃̃o da POPULAÇÃO de ESTUdO}

Todos os pacientes analisados $(\mathrm{n}=40)$ já haviam sido internados devido à LLA e, no período de aplicação dos testes sensoriais, nenhum deles apresentou anemia ou alguma intercorrência como mucosite, gripe, tosse, febre ou outras doenças.

Houve uma distribuição homogênea quanto ao sexo, sendo 21 meninos $(52,5 \%)$ e 19 meninas (47,5\%). Dos pacientes analisados, $47,5 \%$ recebiam tratamento em uma única instituição (ITACI).

\section{ANÁLISE SENSORIAL}

\section{Teste de limiar dos gostos básicos (Threshold)}

Observou-se, tanto no sexo masculino quanto no feminino, que os indivíduos apresentaram maior detecção nas concentrações baixas para todos os gostos básicos, principalmente o ácido para os meninos e o salgado para as meninas.

A Tabela 1 apresenta os valores obtidos entre as duas medidas dos limiares, uma vez que o teste de Threshold foi aplicado em duplicata.

Tabela 1. Coeficientes de correlação intraclasse estimados entre as duas medidas dos limiares

\begin{tabular}{ccc}
\hline $\begin{array}{c}\text { Gostos } \\
\text { básicos }\end{array}$ & $\begin{array}{c}\text { Coeficiente de } \\
\text { correlação } \\
\text { intraclasse }\end{array}$ & $\begin{array}{c}\text { Intervalo com 95\% } \\
\text { de confiança }\end{array}$ \\
\hline $\begin{array}{c}\text { Doce } \\
\text { Salgado }\end{array}$ & 0,6692 & {$[0,4570 ; 0,8098]$} \\
Ácido & 0,9640 & {$[0,9334 ; 0,9808]$} \\
Amargo & 0,8460 & {$[0,7288 ; 0,9153]$} \\
\hline
\end{tabular}

\section{Gosto Doce}

Considerou-se, para os valores de limiares individuais detectados, a seguinte classificação: 0,50 a $0,71 \mathrm{~g} / 1$ baixos; 1,06 a 2,83g/l - médios e acima destes, valores altos de limiares de detecção individual para o gosto doce.

De acordo com a Figura 1, independente do sexo, o maior número de crianças detectou o estímulo nas

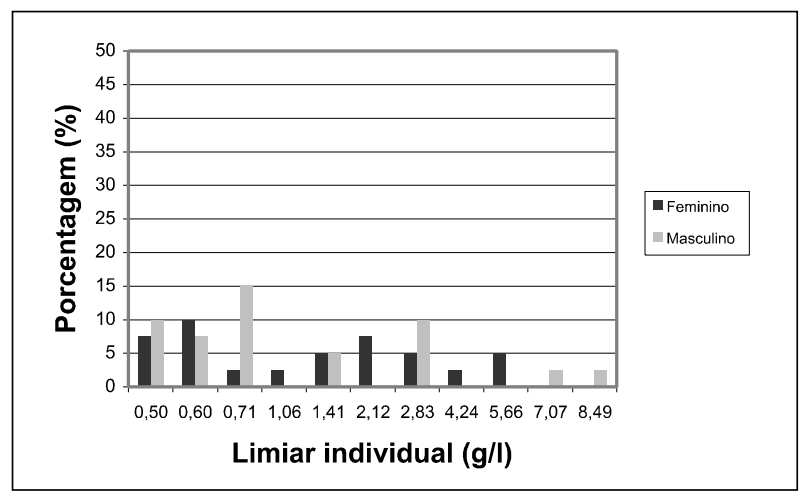

Figura 1. Distribuição dos limiares individuais de detecção do gosto doce, por sexo 
concentrações baixas. As meninas apresentaram-se em maior número entre os limiares baixos e médios e os meninos nos valores baixos.

Estes resultados diferem dos encontrados por Caratin $^{28}$, uma vez que, em sua pesquisa, obteve uma distribuição homogênea entre os sexos. Porém, considerando os limiares mais elevados em ambos os estudos, estes foram observados no sexo masculino.

\section{Gosto Salgado}

Considerou-se, para os valores de limiares individuais detectados, a seguinte classificação: 0,09 a 0,13g/l - baixos; 0,19 a 0,53g/l - médios e acima destes, valores altos de limiares de detecção individual para o gosto salgado.

De acordo com a Figura 2, pode-se observar que, do total das crianças, $32,5 \%$ dos meninos e $25 \%$ das meninas apresentam limiares de detecção baixos. Apenas uma menina detectou o estímulo resultando num limiar mais elevado.

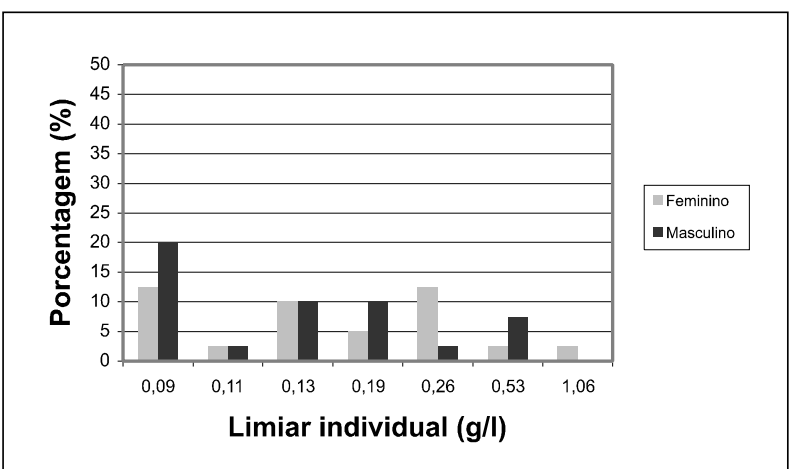

Figura 2. Distribuição dos limiares individuais de detecção do gosto salgado, por sexo

Em trabalho desenvolvido por Caratin ${ }^{28}$, na distribuição segundo os limiares para o gosto salgado de acordo com o sexo, o feminino apresentou maior número nos valores baixos, enquanto no presente estudo foi observado para os indivíduos do sexo masculino. Em ambos os trabalhos, apenas um indivíduo do sexo feminino apresentou o limiar de detecção em valor mais elevado.

Este fato pode indicar que, enquanto as meninas saudáveis são mais sensíveis ao salgado, são os meninos com LLA que apresentam esta característica.

\section{Gosto Ácido}

Considerou-se, para os valores de limiares individuais detectados, a seguinte classificação: 0 - baixo; 0,01 a 0,04g/ 1 - médios e acima destes, ou seja, $0,07 \mathrm{~g} / \mathrm{l}$, valores altos de limiares de detecção individual para o gosto salgado.
De acordo com Figura 3, pode-se observar uma maior distribuição de acordo com o sexo, em limiares baixos e médios de detecção.

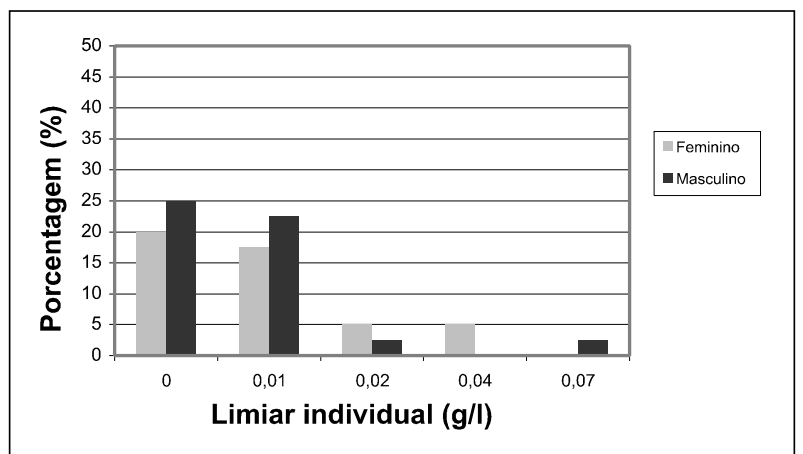

Figura 3. Distribuição dos limiares individuais de detecção do gosto ácido, por sexo

Os resultados obtidos no presente estudo diferem dos de Caratin ${ }^{28}$, uma vez que entre crianças saudáveis foi observado que o maior número de crianças do sexo feminino detectou o limiar em concentrações mais baixas, enquanto entre as crianças portadoras de leucemia linfóide aguda este fato ocorreu para o sexo masculino, indicando maior sensibilidade. Outra diferença foi o fato de haver indivíduos saudáveis de ambos os sexos com limiar mais elevado, enquanto no presente estudo apenas uma criança do sexo masculino demonstrou-se menos sensível ao gosto ácido quando comparada com as demais.

\section{Gosto Amargo}

Considerou-se, para os valores de limiares individuais detectados, a seguinte classificação: 0,03 a 0,05g/l - baixos; 0,07 a $0,14 \mathrm{~g} / \mathrm{l}$ - médios e acima destes $(0,21$ a $0,42 \mathrm{~g} / \mathrm{l})$, valores altos de limiares de detecção individual para o gosto amargo.

A Figura 4 mostra que 30,0\% das meninas e 32,5\% dos meninos apresentaram limiares baixos; $10 \%$ e $15 \%$ médios e $7,5 \%$ e $5 \%$ detectaram nos valores elevados, observando uma distribuição decrescente pelos limiares, em ambos os sexos.

Esses resultados assemelham-se aos obtidos por Caratin $^{28}$, no qual o sexo masculino apresentou maior distribuição pelos limiares de valores baixos, indicando maior sensibilidade ao gosto amargo.

Os resultados obtidos, através da aplicação do teste de limiares para os gostos básicos, de acordo com os parâmetros utilizados, não foram estatisticamente significantes para as variáveis analisadas.

Quando confrontados os valores de limiares obtidos entre as crianças saudáveis e as portadoras de LLA, nota-se que, 
neste último grupo, os valores foram inferiores, indicando que a população do presente estudo possui maior sensibilidade aos gostos básicos do que as crianças saudáveis. Além disso, destaca-se o gosto doce, uma vez que, entre as crianças saudáveis, houve maior concentração nos limiares de valor médio.

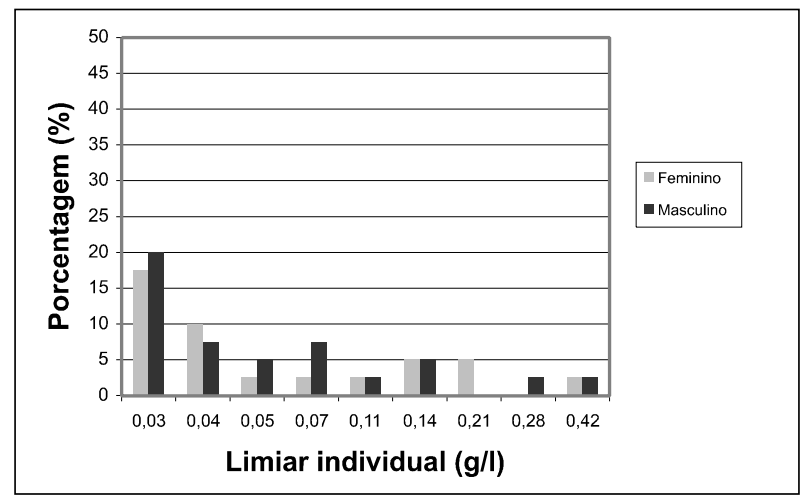

Figura 4. Distribuição dos limiares individuais de detecção do gosto amargo, por sexo

Os meninos apresentaram maior porcentagem nos limiares de valor baixo, indicando maior sensibilidade aos gostos básicos quando comparados às meninas. Porém, quando confrontados com os resultados obtidos por Caratin ${ }^{28}$, nota-se diferença, uma vez que, entre as crianças saudáveis, em relação ao gosto doce, a distribuição foi homogênea em ambos os sexos; quanto ao salgado e ácido, as meninas apresentaram limiares mais baixos; e apenas, quanto ao amargo, os meninos apresentaram maior sensibilidade.

\section{CONCLUSÃO}

A variável sexo não mostrou diferenças significativas em relação à análise dos limiares de detecção dos gostos básicos. Porém, as crianças do sexo masculino mostraram-se mais sensíveis aos gostos básicos do que as do sexo feminino.

Percebe-se a importância da continuidade deste estudo, uma vez que esta população possui características que podem representar especificidades, tais como preferências e aversões alimentares. Além disso, há escassez de trabalhos nessa área em portadores de câncer, o que poderia contribuir para melhorar a aceitação alimentar e a qualidade de vida desta população.

\section{CONSIDERAÇÕES FINAIS}

É constatada grande dificuldade dos pacientes portadores de câncer em se alimentar. A análise sensorial pode ser utilizada como ferramenta para investigar esse agravante.

Optou-se pela leucemia linfóide aguda, por ser o tipo de câncer de maior incidência na infância, até os 15 anos de idade. A menor faixa etária estudada foi determinada a partir da idade em que as crianças são capazes de realizar testes de análise sensorial.

Devem-se realizar pesquisas mais aprofundadas em crianças portadoras de câncer, por possuírem características e necessidades especiais a fim de se estipular parâmetros mais específicos, uma vez que os existentes são direcionados a crianças saudáveis.

A caracterização das crianças portadoras de câncer quanto à sua sensibilidade aos gostos básicos é muito importante para entender sua aceitação e/ou aversão alimentar, pois contribuirá assim para a melhora do estado nutricional e qualidade de vida desta população.

\section{AGRADECIMENTOS}

À FAPESP pela bolsa concedida e às instituições participantes.

\section{REFERÊNCIAS}

1. Teixeira RAP, Bruniera P, Cusato MP, Borsato ML. Câncer infantil. In: Baracat FF, Fernandes HJJ, Silva MJ. Cancerologia atual: um enfoque multidisciplinar. São Paulo: Rocca; 2000:426-51.

2. Vargas PL. Cáncer en pediatria. Aspectos generales. Rev Chil Pediatr. 2000;71(4):1-17.

3. Latorre MRDO. Epidemiologia dos tumores da infância. In: Camargo B, Lopes LF. Pediatria oncológica: noçōes fundamentais para pediatria. São Paulo: Lemar; 2000:7-27.

4. Pedrosa F, Lins M. Leucemia linfóide aguda: uma doença curável. Rev Bras Saúde Matern Infant. 2002; 2(1):63-68.

5. Lopes LF, Mendes WL. Leucemias na infância. In: Camargo B, Lopes LF. Pediatria oncológica: noçōes fundamentais para pediatria. São Paulo: Lemar; 2000:109-18.

6. Lee MLM, Petrilli AS. O tratamento da criança com câncer no Brasil: o debate da migração. Pediatria (São Paulo). 2004;26(1):11-12.

7. GBTLI LLA-99. Protocolo de tratamento da leucemia linfóide aguda em crianças. Sociedade Brasileira de Oncologia Pediátrica. 2000.

8. Cleri LB, Haywood R. Oncology pocket guide to chemotherapy. 5th ed. St Louis: Medical Communications Elsevier Science, 2002.

9. Ferreira RM, Fernandes PLF, Pinheiro LR. Registro de câncer de base populacional: uma proposta para a apresentação de dados pediátricos brasileiros. Rev Bras Cancerol. 1997;43 (2):133-37. 
10.Silverman LB, Sallan SE, Cohen HJ. Treatment of childhood acute lymphoblastic leucemia. In: Hoffman R, Benz EJ, Shatill SJ, Furie B, Cohen HJ, Silberstein LE, et al. Hematology: Basic principles and practice. 3rd ed. Philadelphia: Churchill Livingstone; 2000:1070-1078.

11. Pinho NB, Pacheco SA, Baluz KG, Najman HL, Simma F. Suporte nutricional pediátrico. Rev Bras Cancerol. 1997;43(4):257-63.

12. Garófolo A, Dall'agnol TM, Ancona LF, Novo NF, Juliano Y, Petrilli AS. Ingestão alimentar de crianças e adolescentes com câncer. Anais do VI Congresso Brasileiro de Oncologia Pediátrica; de 20 a 22 agosto 1998; Guarujá (SP). p. 67.

13. Garófolo A, Lopez FA. Novos conceitos e propostas na assistência nutricional da criança com câncer. Rev Paul Pediatr. 2002;20(3):140-46.

14. Lucas B. Nutrição na infância. In: Mahan LK, Escott-Stump S. Alimentos, nutrição e dietoterapia. 9 ed. São Paulo: Rocca; 1998:259-78.

15. Broeder den E, Lippens RJJ, Hof van't MA, Tolboom JJM, Staveren van WA, Hofman Z, et al. Effects of naso-gastric tube feeding on the nutritional status of children with cancer. Eur J Clin Nutr. 1998;52(7):494-500.

16. Mattes RD, Kare MR. Nutrition and chemical senses. In: Shils ME, Shike M, Ross AC, Caballero B. Modern nutrition in health and disease. v.1. 8th ed. Pensylvania: Lea \& Febiger. 1994:524-36.

17. Birch LL. O padrão de aceitação do alimento pelas crianças. In: Anais Nestlé: Sabor e Saciedade. 1999;57:12-20.

18. Moreira APRF, Ishihara CA, Ikemori EHA. O papel do nutricionista no tratamento oncológico. In: Baracat FF, Fernandes HJJ, Silva MJ. Cancerologia atual: um enfoque multidisciplinar. São Paulo: Rocca. 2000:186-97.

19. Schiffman SS. Fisiologia do paladar. In: Anais Nestlé: Sabor e saciedade. 1999;57:1-11.
20.Associação Brasileira de Normas Técnicas (ABNT). Análise sensorial de alimentos e bebidas. NBR 12806. São Paulo; 1993.

21. Monteiro CLB. Técnicas para a avaliação sensorial de alimentos. 2a ed. Curitiba: Ceppa; 1984.

22. Moraes MAC. Método para avaliação sensorial dos alimentos. 6a ed. Campinas: UNICAMP; 1988.

23. Associação Brasileira de Normas Técnicas. Teste de sensibilidade em análise sensorial. NBR 13172. São Paulo; 1994.

24. Dutcosky SD. Análise sensorial de alimentos. Curitiba: Champagnat; 1996.

25. Chaves JBP, Sprosser RL. Práticas de laboratório de análise sensorial de alimentos e bebidas. Universidade Federal de Viçosa: Imprensa universitária; 1993.

26. James CE, Laing DG, Oram N. A comparison of the ability of 8-9-year-old children and adults to detect taste stimuli. Physiol Behav. 1997;62(1):193-97.

27. Liem DG, Mars M, GraafC. Consistency of sensory testing with 4 and 5 year-old children. Food Quality and Preference. 2004;15:541-48.

28. Caratin CVS. Análise dos limiares de detecção dos gostos básicos e sensibilidade ao 6-n-propiltiouracil em crianças de 7 a 10 anos. [Dissertação de Mestrado]. São Paulo: Universidade de São Paulo; 2004.

29. Woods MP. Taste and flavor perception. Proc Nutr Soc. 1998;(56):603-607.

30. International Organization for Standartization. Sensory analysis - methodology - flavor profile methods. ISSO 6564. Switzerland; 1985.

31. Statistical Package for the Social Science. SPSS for Windows: versão 10.0. Illinois: SPSS Incorporation; 2000.

32. Conover WJ. Practical nonparametric statistics. 3rd ed. New York: John Wiley \& Sons; 1998.

\section{Abstract}

Childhood cancer is defined as any malignant neoplasm occurring in individuals under fifteen years of age. Leukemia is the most common type in this group. Acute lymphocytic leukemia (ALL) occurs more often in boys than in girls, with a ratio of 1.2:1. Approximately $50 \%$ of pediatric cancer patients report eating disorders, including loss of appetite, altered taste, nausea, vomiting, and diarrhea, among others. Determination of detection thresholds for basic tastes helps understand the aversion to food and eating preferences in children with cancer. The purpose of the current study was to identify the detection thresholds for basic tastes according to the gender of children with ALL on chemotherapy. Children from three oncological institutions in São Paulo participated: ITACI/ICR/HC/ FMUSP, IOP-GRAAC, and the A. C. Camargo Hospital. The sample included 40 children from 6 to 15 years of age, (21 males and 19 females), following the GBTLI 99 treatment protocol. The sensitivity threshold test was applied, using paired samples of six increasing concentrations for each taste. Non-parametric tests were applied using the SPSS program. No association was observed between gender and detection threshold for basic tastes. Key words: Children, Leukemia, Threshold, Gender 